\title{
Nosocomial Infections in a Tertiary Healthcare Center in Lebanon: Optimization of Safety Protocols
}

\author{
Pamela Abdallah, Clara Chaiban, Raymond El-Hajj, Claude Afif, and Elias Chalhoub
}

\section{ABSTRACT}

\begin{abstract}
Objective: The Saint George Hospital - University Medical Center (SGHUMC)- in Ashrafieh, Lebanon - aims at achieving excellence in medical services by empowering the Infection Control and Prevention Department (ICPD) to maintain a healthy environment and ensure the safety of patients and medical personnel equally. In association with the University of Balamand, two interventions were employed to attempt at further ameliorating the ICPD and the patient safety.
\end{abstract}

Interventions: The first intervention constitutes of a cost-benefit analysis to evaluate the possibility of substituting the use of reusable gowns with disposable ones and the second is a questionnaire allowing the evaluation of the physician satisfaction with the ICPD at SGH-UMC.

Results: The cost-benefit analysis suggested that the use of reusable gowns was more cost effective than the use of disposable gowns even after taking into consideration the attributable cost of nosocomial infections. The physician's satisfaction questionnaire highlighted the physician's various perceptions regarding the ICPD and the infection control and prevention in the hospital and recommended some corrective actions to overcome the obstacles faced.

Conclusion: The process of quality improvement requires the employment of several quality tools, identifying the problems and attempting to solve them; therefore, development and distinction can only be attained after a process of self-evaluation, troubleshooting and corrective actions.

Keywords: Disposable Gowns, Infection Control and Prevention, Patient Safety, Reusable Gowns.
Submitted : February 8, 2021

Published : February 25, 2021

ISSN: $2593-8339$

DOI: $10.24018 /$ ejmed.2021.3.1.708

P. Abdallah ${ }^{1}$

Department of Medical Laboratory Sciences, Faculty of Health Sciences, University of Balamand, Beirut, Lebanon. (e-mail: pamela_ab@hotmail.com) C. Chaiban ${ }^{1}$

Department of Medical Laboratory Sciences, Faculty of Health Sciences, University of Balamand, Beirut, Lebanon. (e-mail: clarachaiban@ ${ }^{@}$ hotmail.com) R. El-Hajj

Department of Medical Laboratory Sciences, Faculty of Health Sciences, University of Balamand, Beirut, Lebanon. (e-mail: raymond.hajj ${ }^{\circledR}$ balamand.edu.lb) C. Afif

Faculty of Medicine, University of Balamand, Beirut, Lebanon.

Departement of Infection Control, Saint George Hospital-University Medical Center, Beirut, Lebanon

(e-mail: cmafif@stgeorgehospital.org ) E. Chalhoub*

Department of Medical Laboratory Sciences, Faculty of Health Sciences, University of Balamand, Beirut, Lebanon.

(e-mail: elias.chalhoub2@gmail.com)

* Corresponding Author

${ }^{1}$ Authors contributed equally to this work

\section{INTRODUCTION}

Nosocomial Infections occur in 1 out of 10 patients that are admitted to the hospital; they increase the hospitalization cost in addition to morbidity and mortality [1]. Infection control and prevention (ICP) consist of decreasing the incidence of nosocomial infections (NI) or Hospital Acquired Infections (HAI) and reducing the risk of transmission of microorganisms between patients, from patients to healthcare workers, and from healthcare workers to patients [2]. These are the main objectives of the Infection Control and Prevention Department (ICPD) at the Saint George Hospital - University Medical Center (SGH-UMC). The SGH-UMC is a non-profit University Medical Center, founded by the Orthodox Archdiocese of Beirut in 1878 and located in Ashrafieh, Beirut, Lebanon [3].

The SGH-UMC aims to achieve excellence in medical services by implementing healthcare quality standards and employing quality management tools and methods to seek constant improvement and attain the hospital's strategic goals [3].

The ICPD at the SGH-UMC applies policies and protocols related to prevention of NI such as hand hygiene, cleaning, disinfection and sterilization, immunization of the staff, prevention and control of outbreaks, linen reprocessing and laundry, and a well-planned surveillance program designed to monitor and control all the information from various hospital units and departments. In summary, it attempts to control and prevent NI, to educate and train the involved parties (healthcare workers, patients, and caregivers) of regular infection control practices, to develop guidelines for work practices in health care facilities, to implement recommended infection control and prevention measures, to control the emergence and transmission of infectious pathogens, to 
ensure the cleanliness of the environment with the use of proper disinfectants, to ensure the implementation of appropriate disinfection and sterilization procedures and products, and to ensure that all the staff, including medical doctors and nurses, overcome reluctance to change old procedures and habits and adopt new recommended procedures as for international evidence-based guidelines.

Hospital Acquired Infections or Healthcare Associated Infections (HAI) are infections that occur after 48 to 72 hours of hospital admission, 10 days after discharge, and/or 30 days after an operation, the patient develops the infection during hospitalization; the pathogen is neither present nor incubating upon hospital admission [1], [4], [5]. HAI include, but are not limited to, surgical-site infections, ventilator-associated pneumonia, catheter-associated urinary tract infections and central line-associated bloodstream infections [4].

HAI are often associated with an abuse of antimicrobial agents, resistance, and cross-contamination of the pathogen among patients [4]. This results in a prolonged hospital stay and an increase in morbidity and mortality which are associated with an increased hospitalization cost as well as a marred image and a distorted reputation among competitors in the healthcare market [5]. Therefore, maintaining the patients' health and aiming at providing optimum healthcare services have become the basis of treatment at SGH-UMC.

The ICPD follows patients, personnel, and logistics. It surveys and monitors each patient admission and discharge diagnosis, patient's file, surgical procedures, and the administered medications. It surveys hospital personnel to ensure abidance to ICP policies and protocols. It also surveys water and air systems to prevent contamination with dust, pathogens, etc.

HAI are generally contained by proper hand and body hygiene, abiding to equipment sterilization protocols, and following the ICPD recommendations [1].

The goal of the study and interventions in the ICPD at SGH is to fill in the gaps of quality improvement, technology implementation, and process improvement of the department. The study consists of 2 interventions: a cost benefit analysis of amendments to the protocol of Standard Precautions and Isolation Precautions to propose a shift from reusable linen gowns to the use of disposable gowns, and the evaluation of physician's satisfaction and awareness regarding the ICP, its protocols and guidelines and implementation.

\section{INTERVENTIONS}

\section{A. Intervention 1}

A cost benefit analysis was conducted to evaluate the advantages and disadvantages of shifting from reusable to disposable gowns. Morning rounds were done on every floor that has standard and isolation precautions. The floors involved included the third floor (Intensive Care Unit and Cardiac Care Unit), fourth floor (Pediatrics, Pediatric Intensive Care Unit, Neonatal Intensive Care Unit), sixth floor (Oncology for Neutropenic patients), seventh and eighth floor for isolated rooms only, and 9th floor (Oncology for Pediatrics, Neutropenic patients and Neurosurgery, Psychiatry and Geriatrics isolated patients). Healthcare practitioners and patient visitors were observed for 2 weeks to assess whether they were abiding by ICP guidelines and recommendations (use of gowns, gloves... upon every entry to patient rooms).

The step-by-step method was done as follows:

- Morning rounds were done on the third, fourth, sixth, seventh, eighth and ninth floors where a paper checklist was placed on every isolated room.

- For each entry, visitors as well as healthcare workers including registered nurses, practical nurses, residents and physicians were asked to put a check mark for each time they enter a patient room for visits or for administration of medications or procedures, respectively.

- Papers were changed every twenty-four hours for a duration of 2 weeks.

The cost of using disposable gowns was evaluated using the above collected information and the cost of using reusable gowns was calculated after visiting the laundry department. The costs and benefits were compared, and a detailed analysis was performed.

\section{B. Intervention 2}

Physicians' satisfaction, awareness, and compliance with ICP guidelines and recommendations were evaluated via a Physician's Satisfaction Questionnaire that was addressed to all the physicians at SGH-UMC. The questionnaire was distributed to 170 physicians to be filled in a margin of three weeks. The answers to the simplified questionnaire of thirteen items related to the above-mentioned matters in infection control and prevention were then entered into an Excel sheet and analyzed.

\section{RESUlts}

\section{A. Intervention 1}

The number of entries to the patients' rooms by healthcare workers and patient visitors was evaluated to allow the assessment of the average number of disposable gowns that will be used.

TABLE I: THE NUMBER OF ROOMS PER DEPARTMENT REQUIRING THE USE OF DISPOSABLE GOWNS

\begin{tabular}{cc}
\hline \hline Floor & Patient Rooms \\
\hline $3^{\text {rd }}$ Intensive Care Unit (ICU) & 10 \\
$3^{\text {rd }}$ Cardiac Care Unit (CCU) & 1 \\
$3^{\text {rd }}$ Cardiac Surgery Unit (CSU) & NONE \\
$4^{\text {th }}$ Pediatric and Neonatal ICU (PICU and NICU) & 15 \\
$4^{\text {th }}$ Pediatrics (Average Isolated Patients) & 2 \\
$6^{\text {th }}$ and $9^{\text {th }}$ Neutropenic (Average Isolated Patients) & 4 \\
$7^{\text {th }}$ and $9^{\text {th }}$ Isolated (Average Isolated Patients) & 4 \\
$8^{\text {th }}$ Isolated (Average Isolated Patients) & 6 \\
Total & 42 \\
\hline \hline
\end{tabular}


TABLE II: THE EVALUATION OF EXPENSES OF USING Two DIFFERENT TYPES OF GOWNS

\begin{tabular}{|c|c|}
\hline \multicolumn{2}{|c|}{ Disposable Gowns } \\
\hline $\begin{array}{l}\text { Average gowns per patient } \\
\text { per day }\end{array}$ & 25 gowns \\
\hline Average cost per gown & 0.5 cents \\
\hline Average total gowns per day & $\begin{array}{c}25 \text { gowns per room } \mathrm{x} 42 \text { rooms }=1050 \\
\text { gowns }\end{array}$ \\
\hline Average total gowns per year & $\begin{array}{c}1050 \text { gowns per day } x \text { } 365 \text { days }=383,250 \\
\text { gowns }\end{array}$ \\
\hline Reusable Gowns & $\begin{array}{l}383,250 \text { gowns } \times 0.5 \text { cent }=191,625 \text { USD } \\
\text { usable Gowns }\end{array}$ \\
\hline $\begin{array}{l}\text { Average cost of washing } 1 \\
\text { cycle of white dirty linen }\end{array}$ & 11.5 USD per $180 \mathrm{~kg}$ \\
\hline $\begin{array}{l}\text { Average cost of related } \\
\text { consumption (water, } \\
\text { electricity, labor, steam, } \\
\text { vapor...) }\end{array}$ & 20 USD \\
\hline Weight of 1 gown & $450 \mathrm{~g}$ \\
\hline Number of gowns per load & $180 \mathrm{~kg} / 0.45 \mathrm{~kg}$ per gown $=400$ gowns \\
\hline Cost of washing 400 gowns & 11.5 USD + $20 \mathrm{USD}=11.5 \mathrm{USD}$ \\
\hline Cost of a new gown & 16 USD \\
\hline $\begin{array}{l}\text { Average duration of use of } \\
\text { every gown }\end{array}$ & 7 months \\
\hline
\end{tabular}

A cost benefit analysis was applied to determine whether the implementation of this intervention is feasible at the SGHUMC. The analysis would allow the comparison of the total expected cost of each option against its total expected benefits to assess whether the benefits outweigh the costs and by how much.

The formula recruited for this analysis is the following:

$$
\text { Cost }=\frac{\text { Cost of } 1 \text { Gown } x \text { Average Gowns per Day }}{\text { Average Number of months of }}
$$

Therefore, the cost of using reusable gowns would equal 2,400 USD per month,

$$
\left(\operatorname{Cost}=\frac{16 \times 1050}{7}\right)
$$

whereas the cost of using disposable gowns would equal 15,969 USD per month.

$$
\left(\text { Cost }=\frac{0.5 \times 365 \times 1050}{12}\right)
$$

Thus, the cost of using disposable gowns is much higher than the sum of the cost of using reusable gowns and the attributable cost of NI.

\section{B. Intervention 2}

Out of the 170 sent questionnaires, only 30 answers were received accounting for $17.6 \%$ of the target population. Among the 30 received answers, 3 doctors preferred not to specify which specialty they are in and the 27 others came from medical doctors in various specialties like general pediatrics, pediatrics hematology/oncology, gastroenterology pediatrics, nephrology, neurology, psychiatry, infectious diseases, gastroenterology, laboratory medicine, family medicine, pulmonary, general surgery, cardiothoracic surgery, vascular surgery, orthopedic surgery, urology surgery, obstetrics and gynecology, and ophthalmology surgery.
Reasons for not answering the questionnaire included lack of time, being abroad or losing the questionnaire altogether at the secretary's office.

According to the responding physicians' answers to the questionnaire Appendix 1, almost half the physicians are unaware of the availability of the ICP manual at the hospital although the vast majority of them are satisfied with the implementation of ICP practices and agree that the ICPD staff are always available to answer any questions. Despite their satisfaction with the ICPD, $83 \%$ of the responding physicians believe that better enforcement of the ICP recommendations is still required to further decrease the rates of NI. Some recommendations and policies are controversial, like the restriction of antibiotic intake policy that aims at limiting bacterial resistance. $70 \%$ the responding physicians agree to this specific policy whereas $13 \%$ disagree and $17 \%$ are neutral towards it.

The responding physicians' opinions regarding reminders and means of implementing ICP recommendations and guidelines vary greatly whereby some stated that enough reminders are available whereas others disagreed, and a few did not have an opinion regarding the matter; therefore, manifesting a need in better placing or modifying the reminders to make them more visible.

The responding medical doctors agree in general that an immunization program for all hospital personnel is a necessity to limit the toll of infectious diseases and more than half of them are aware of their own current immunization status. However, this also proves that not all the physicians are treating their safety with utmost importance and some of them are treating the immunization plan lightly.

$60 \%$ of the responding physicians confirm the presence of an active multi-disciplinary ICP committee that includes four physicians whereas the rest deny it or are unaware of its presence altogether. Therefore, there is a gap regarding the perception and exposure of practicing physicians to this committee.

Physicians were also asked about the discussion of ICP and patient safety in their regular meetings where the majority of them stated that this is rarely done. When asked about the predominant categories of NI, answers varied among physicians yet the most common were Central LineAssociated Bloodstream Infections (CLABSI) and Lower Respiratory Tract Infections (LRTI) followed by Urinary Tract Infections (UTI) and Surgical-Site Infections (SSI).

Most of the responding physicians confirm the presence of hand hygiene posters and alcohol dispensers around the hospital. They also claim that the performance of infection control is commonly or partially done where they stated that regular feedback and updates regarding patients as well as regarding microorganism susceptibility are performed yet they almost unanimously answered that regular testing for tuberculosis is not performed.

Physicians answers regarding corrective measures to reduce HAI were grouped into 8 major categories. 1) CAUTI Protocol: the time of Foley catheter insertion should be recorded in the chart and a reminder should be set up. 2) Discussions: infection control and patient safety issues should be discussed more often, efficiently, and regularly at meetings to increase physicians' awareness and to better communicate, among physicians, errors that have occurred 
with patients and families. This would also encourage and promote preventive and corrective measures for patients, staff and visitors. 3) Education: Frequent education is recommended among physicians, nurses and hospital staff as well as raising awareness among patient families concerning ICP and patient safety. 4) Approach by protocol and guidelines: the application of worldwide tested studies with proven results regarding measures, protocols and bundles being unnecessarily delayed from a medical standpoint (such as Ventilator Associated Pneumonia (VAP) bundle), packaged nutrition, proper isolation measures (such as 1:1 ratio for Intensive Care Unit patients), Intensive Care Unit bundle... Physicians recommended some interventions that will improve patient outcome when used collectively. 5) Hand Hygiene Compliance: Hand hygiene should be more implemented by interns and residents especially before and after changing dressings in addition to increasing the frequency and availability of alcohol-based solution and foam dispensers in front of each patient room. 6) Better use of PPE: The better use of PPE such as gloves, masks, hair net, cover shoes, and gowns especially in the operating rooms and in the Isolating Rooms in addition to the usage of disposable gowns in the Intensive Care Unit to reduce the rate of NI. 7) Antimicrobial Stewardship: the reduction of antibiotic use was recommended in the case where antibiotic administration is not a to limit bacterial resistance to antibiotics. 8) Immunization and PPD test: PPD tests for Tuberculosis should be done periodically once per year.

\section{DISCUSSION}

\section{A. Intervention 1}

The cost of the disposable gowns is higher than the cost of reusable gowns plus the nosocomial infection attributable cost.

The cost of washing reusable gowns is lower than the cost of buying disposable gowns. The manager of the Laundry Department would need to supply the hospital with 500 additional gowns instead of 150 gowns as an average. Therefore, the reusable gowns are going to be used once by the Healthcare workers and visitors, and then will be discarded to the laundry room for washing. Hence, an alternative plan is to use the reusable gowns as single use for one entry instead of multiple entries per day for each isolated patient. It is important to note that in addition to a lower cost attributed to reusable gowns, they also perform similarly to disposable ones when it comes to patient safety and comfort of use [6], [7]; they also have an advantage when it comes to the environmental impact [8].

\section{B. Intervention 2}

A gap of communication is present between the ICPD and the physicians. This is further assured by the fact that only 30 questionnaires were returned even though the questionnaire was approved and signed by the Medical Director of the hospital.

To increase the awareness regarding the importance of Infection Control, Prevention, and Patient safety at the hospital and increase implementation of protocols, guidelines and physicians' satisfaction regarding ICP, an education day should be implemented at least once per month. All the lectures of Infection Control and Prevention should be addressed, and all the physicians should be encouraged to attend the lectures to reinforce current standards of infection control and prevention practices, emphasizing the importance of PPE. Hence, an effective Infection Control and Prevention Program should collaborate with patient safety, quality assurance, and occupational health.

Furthermore, hand hygiene should be improved where it should play a main role to reduce and prevent HAI through: 1) adequate supply of alcohol-based solution bottles to improve the adherence to hand hygiene by all medical staff, 2) alcohol-based solution bottles should be replaced immediately when empty next to patient care and the presence of alcohol-based solution at the patient care, and 3) education sessions related to hand hygiene should be obligatory to all the hospital staff including physicians, residents, and interns.

Moreover, Ventilator Associated Pneumonia should be reduced and prevented through the application of the VAP bundle with the needed items and materials and ensuring the availability of mouth hygiene and subglottic secretion aspiration. Furthermore, Center Venous Catheter (CVC) related infections should be also reduced and prevented through insurance of aseptic techniques during insertion of $\mathrm{CVC}$, and standardization of insertion and management of protocols.

Therefore, physicians are responsible of 1) protecting and giving care for their patients from other infected patients and from the hospital staff, 2) complying with the practices and recommendations that are approved by the Infection Control and Prevention Committee, 3) obtaining the right cultures when there is a suspension of an infection, and 4) advising staff, patients, and their relatives on practices and procedures to prevent transmission of infections. It is one important responsibility of the physician in the hospital to deliver direct patient care to reduce infection and respect all the protocols and practices that are related to prevention such as hand hygiene, cleaning, disinfection and sterilization, and immunization. The cooperation between the Infection Control and Prevention staff and physicians at the hospital will help and aid in increasing the awareness of Infection Control, Prevention, and Patient safety, and therefore, by implementing the protocols and guidelines.

\section{CONCLUSION}

The traditional view of health is changing. Health is no longer considered to be dependent on medical care alone and the simple absence of disease. Today the prevailing opinion is that optimal health can generally be achieved through environmental safety, organizational changes, and ameliorated lifestyles.

Based on all the above here are some recommendations that should be taken into consideration:

- Implementation of a new software at the ICPD: This can decrease the staff's lost time by eliminating valueless administrative tasks and can allow staff to spend more time on education programs and monitoring infection rates, trends and sharing data more often.

- Education and training programs: Raising awareness among families, residents, interns, and nurses regarding 
Infection Control, Prevention, and Patient Safety.

- ICPD lacking adequate number of personnel: The presence of an additional Analyst for daily data entry and analysis will help the available staff in fulfilling their daily tasks without being overwhelmed by work.

- Holding conferences and lectures in case of possible outbreaks: Whenever the rate of infection increases, conferences and lectures should be held for interns, residents, and physicians with the Infection Control Committee for awareness regarding HAI.

- Awareness and implementation regarding the use of PPE: Using disposable gowns, gloves, hairnet, shoe cover, and masks in the Intensive Care Unit and isolated rooms.

- Improvement of communication: Better communication between ICPD with physicians to increase awareness of ICP to implement protocols and guidelines

- Better financing: Finding budgets to buy and supply hospitals with PPE such as reusable gowns and hand hygiene bottles..

\section{APPENDIX}

Appendix 1:
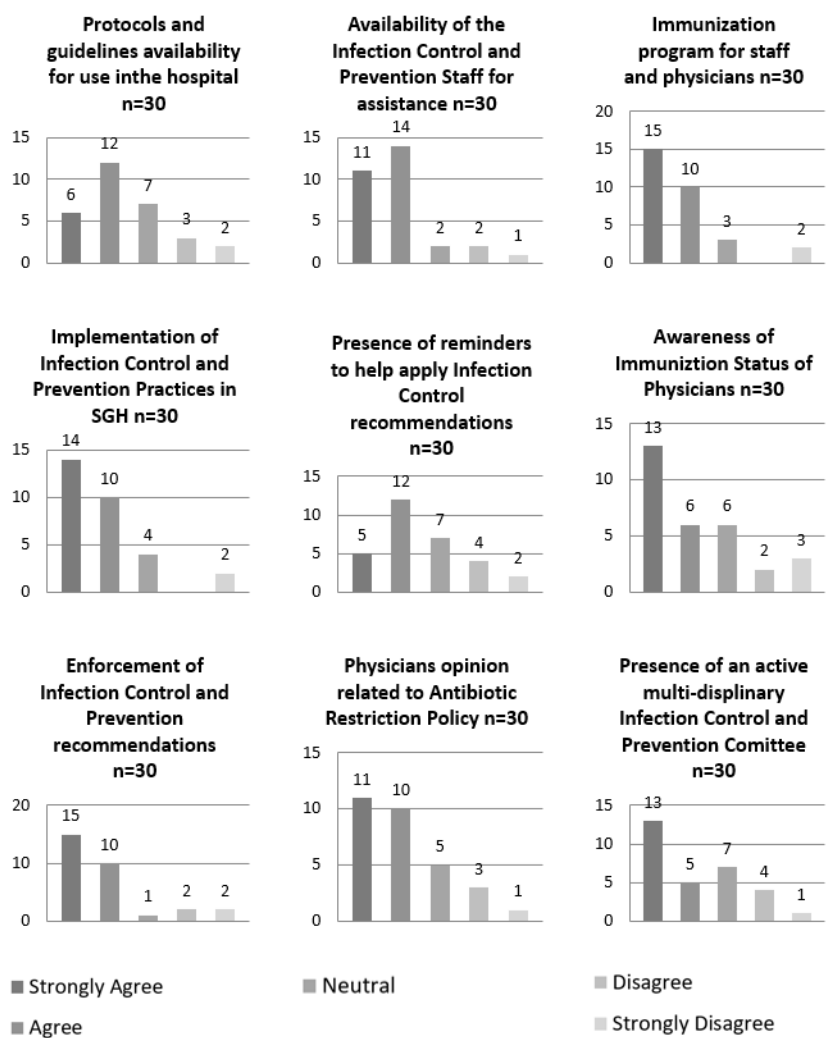

- Strongly Agree

agree
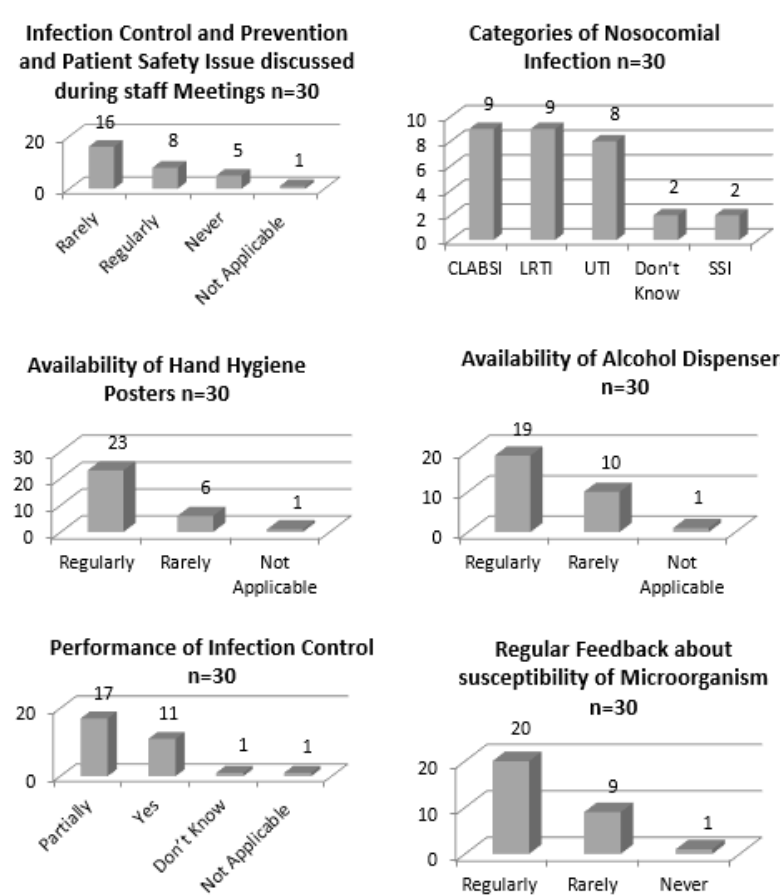

Regular Updates and Feedbacks $\mathrm{n}=\mathbf{3 0}$
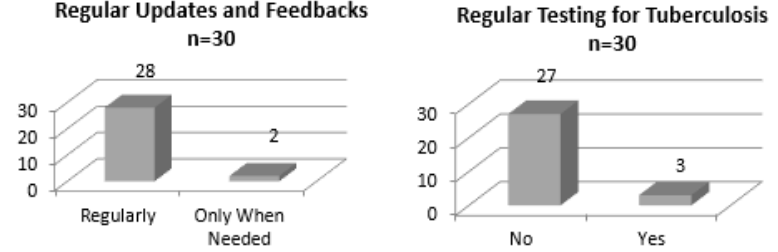

\section{ACKNOWLEDGMENT}

Authors would like to deeply thank the SGH-UMC administration and staff, especially the ICPD staff, for their contribution and their time that allowed this project to be a successful one. We would like to express our gratitude to Ms. Mona Haddad, who despite her busy schedule took time to help and engage in this project. The authors would also like to thank Dr. Nadim Karam, founder Dean of the FHS for his full support.

\section{REFERENCES}

[1] A. Revelas, "Healthcare - Associated Infections: A Public Health Problem”. Nigerian Medical Journal, 53(2):59-64, April 2012.

[2] World Health Organization, "Infection Prevention and Control". Accessed from: https://www.who.int/gpsc/ipc/en/. 2019.

[3] Saint George Hospital University Medical Center, "About Us" Accessed from: https://www.stgeorgehospital.org/aboutus. 2019.

[4] Centers for Disease Control and Prevention, "Healthcare-Associated Infections". Accessed from: https://www.cdc.gov/hai/. 2019.

[5] Cavalcante EFO, Pereira, I. R. B. O., Leite, M. J. V. F., Santos AMD, Cavalcante CAA. "Implementation of patient safety centers and the healthcare-associated infections". The Revista Gaucha de Enfermagem; 40:e20180306. 2019.

[6] Overcash M. "A Comparison of Reusable and Disposable Perioperative Textiles: Sustainability State-of-the-Art". Anesthesia and Analgesia;114(5):1055-1066. 2012.

[7] McQuerry M, Easter E, Cao A. "Disposable versus reusable medical gowns: A performance comparison". American journal of infection control. October 2020.

[8] Vozzola E, Overcash M, Griffing E. "Environmental considerations in the selection of isolation gowns: A life cycle assessment of reusable and disposable alternatives". American journal of infection control; 46(8):881-886. August 2018. 